\title{
Effect of disorder on shrinkage-induced fragmentation of a thin brittle layer
}

\author{
Zoltán Halász, ${ }^{1}$ Akio Nakahara, ${ }^{2}$ So Kitsunezaki, ${ }^{3}$ and Ferenc Kun ${ }^{1,4, *}$ \\ ${ }^{1}$ Institute for Nuclear Research, Hungarian Academy of Sciences (Atomki), P.O. Box 51, H-4001 Debrecen, Hungary \\ ${ }^{2}$ Laboratory of Physics, College of Science and Technology, Nihon University, 7-24-1 Narashinodai, Funabashi 274-8501, Japan \\ ${ }^{3}$ Research Group of Physics, Division of Natural Sciences, Faculty of Nara Women's University, Nara 630-8506, Japan \\ ${ }^{4}$ Department of Theoretical Physics, University of Debrecen, P.O. Box 5, H-4010 Debrecen, Hungary
}

(Received 8 May 2017; published 25 September 2017)

\begin{abstract}
We investigate the effect of the amount of disorder on the shrinkage-induced cracking of a thin brittle layer attached to a substrate. Based on a discrete element model we study how the dynamics of cracking and the size of fragments evolve when the amount of disorder is varied. In the model a thin layer is discretized on a random lattice of Voronoi polygons attached to a substrate. Two sources of disorder are considered: structural disorder captured by the local variation of the stiffness and strength disorder represented by the random strength of cohesive elements between polygons. Increasing the amount of strength disorder, our calculations reveal a transition from a cellular crack pattern, generated by the sequential branching and merging of cracks, to a disordered ensemble of cracks where the merging of randomly nucleated microcracks dominate. In the limit of low disorder, the statistics of fragment size is described by a log-normal distribution; however, in the limit of high disorder, a power-law distribution is obtained.
\end{abstract}

DOI: 10.1103/PhysRevE.96.033006

\section{INTRODUCTION}

Layers of heterogeneous materials attached to a substrate often undergo sequential cracking due to shrinkage stresses caused by desiccation, mismatch of thermal expansion coefficients, or by stretching or compression of the substrate. From the spectacular crack patterns of dried out lake beds through the polygonal ground patterns of permafrost regions to the formation of columnar joints in cooling volcanic lava, shrinkage-induced cracking is responsible for a large variety of complex crack structures in nature [1,2].

Under laboratory conditions, shrinkage-induced cracking is usually investigated by desiccating thin layers of dense colloidal suspensions in a container. Experiments performed with suspensions of coffee powder [3], clay [4,5], and calcium carbonate [6] have revealed polygonal crack patterns with a high degree of isotropy of the crack orientation. Along the cellular crack network the thin layer breaks up into pieces such that the evolution of the crack pattern is usually characterized by the average size (linear extension, area, mass) of fragments, by the evolution of the mass distribution of fragments [1], and by the degree of opening of the cracks [5] as function of the shrinkage strain or time.

It is of great interest how to control the structure of shrinkage-induced two-dimensional crack patterns $[1,6,7]$ also due to its high potential for technological applications $[8,9]$. Recently, it has been demonstrated experimentally for dense calcium carbonate and magnesium carbonate hydroxide pastes that applying mechanical excitation by means of vibration or flow of the paste the emerging desiccation crack pattern remembers the direction of excitation [6,7]: for vibration and flow the primary crack orientation becomes perpendicular and parallel to the direction of excitation, respectively. Detailed experiments varying the way of excitation, furthermore, the strength and range of interaction of colloidal particles have

\footnotetext{
*ferenc.kun@science.unideb.hu
}

revealed that the memory effect of pastes provides an efficient way of controlled generation of crack patterns in thin layers. For example, by applying vertical vibration to the thin layer of paste, the position of crack formation can be controlled, because Faraday waves localize stronger horizontal vibration at node regions where cracks appear earlier than in other regions [10]. Effectiveness of memory of paste can be controlled by adding sodium chloride to paste, because the strength and range of interaction between charged colloidal particles in paste can be controlled by the screening effect [11]. Controlled crack patterning has been demonstrated to provide opportunities for future applications, e.g., to fabricate high-performance electrochromic structures $[8,9]$.

The position and orientation of cracks are determined by the local stress field induced by shrinkage and by the disorder of the material. In colloidal suspensions the amount of disorder can be controlled, e.g., by varying the range and the functional form of the size distribution of colloid particles. Vibration and flow of pastes gives rise to well-ordered crack structures since the initial mechanical excitation imprints a directional or spatial distribution of density fluctuations and plastic deformation, which overcomes the effect of disorder in the local fracture strength for crack formation $[10,12]$. Hence, it is an interesting question how the competition of the structural heterogeneity and the disorder of the local fracture strength of the material determines the evolution of shrinkage-induced cracking and the size distribution of fragments.

In the present paper, we address this question in the framework of a discrete element model of a brittle layer of heterogeneous materials attached to a substrate. The model material has two sources of disorder, namely, structural and strength disorder introduced by the underlying random lattice of discretization and by the random breaking thresholds of cohesive elements, respectively. Varying the amount of strength disorder at a fixed degree of structural heterogeneity we investigate the dynamics of breakup and the resulting size distribution of pieces. As the most interesting outcome our 
computer simulations revealed a transition in the cracking mechanism which gives rise to log-normal and power-law mass distributions of fragments at low and high disorder.

\section{DISCRETE ELEMENT MODEL OF A THIN BRITTLE LAYER ON A SUBSTRATE}

We introduce a simple discrete element model (DEM) of a thin brittle layer attached to a rigid substrate. The model is essentially based on a DEM of heterogeneous brittle materials, which has proven successful in studying the dynamic fragmentation process induced by impact or explosion [13-16]. In the following, we briefly summarize the main steps of the model construction and highlight how it is adopted to the problem of shrinkage-induced fragmentation.

\section{A. Discretization of the layer}

In the model a rectangularly shaped layer of side length $L$ is considered. The layer is discretized in terms of randomly shaped convex polygons, which are obtained by a Voronoi tessellation of the rectangle $[13,15]$. For the construction of Voronoi polygons we apply the so-called vectorizable random lattice which makes possible to control the degree of structural disorder [17]: first a regular square lattice is placed on the layer with a lattice spacing $l=1$ which defines the unit length of the system. Inside each plaquette of the lattice a smaller square is centered with a side length $a$ which can assume values in the range $0 \leqslant a \leqslant l$. The basis points of the Voronoi construction are determined such that in each small square a random point is thrown independently of the others. Using this discretization strategy the layer is decomposed into space filling convex polygons where the degree of structural disorder is controlled by the value of $a$ : in the limit of $a=0$ a regular square lattice is obtained while increasing side length $a \rightarrow l$ results in increasing heterogeneity. In the present calculations the value of $a$ was set to $a=0.8$ which provides a random lattice of a high degree of isotropy [15,17]. A representative example of a small system of size $L=30$ is presented in Fig. 1(a). In two-dimensions the polygons have three degrees of freedom, i.e., the two coordinates $\left(x_{i}, y_{i}\right)$ of the center of mass $\vec{r}_{i}$, and the rotation angle $\phi_{i}(i=1, \ldots, N)$, where $N$ denotes the number of particles.

\section{B. Interactions}

To represent the mechanics of the layer the center of mass of polygons, which are nearest neighbors in the initial Voronoi tessellation, are connected by beam elements $[13,15]$. This way a triangular lattice of beams is attached to the polygonal material elements [see Fig. 1(b)]. The geometrical properties of beams depend on the random tessellation such that the initial length $l_{0}^{i j}$ and cross section $S_{0}^{i j}$ of beams are the distance of the centers and of the length of the common side of the connected polygons, respectively.

During the deformation of the system the beams suffer longitudinal deformation, shear, and bending giving rise to forces and torques acting on the polygons. The deformation of a beam is illustrated in Fig. 2. For the beam between sites $i$ and $j$, in the local coordinate system of the beam, the normal
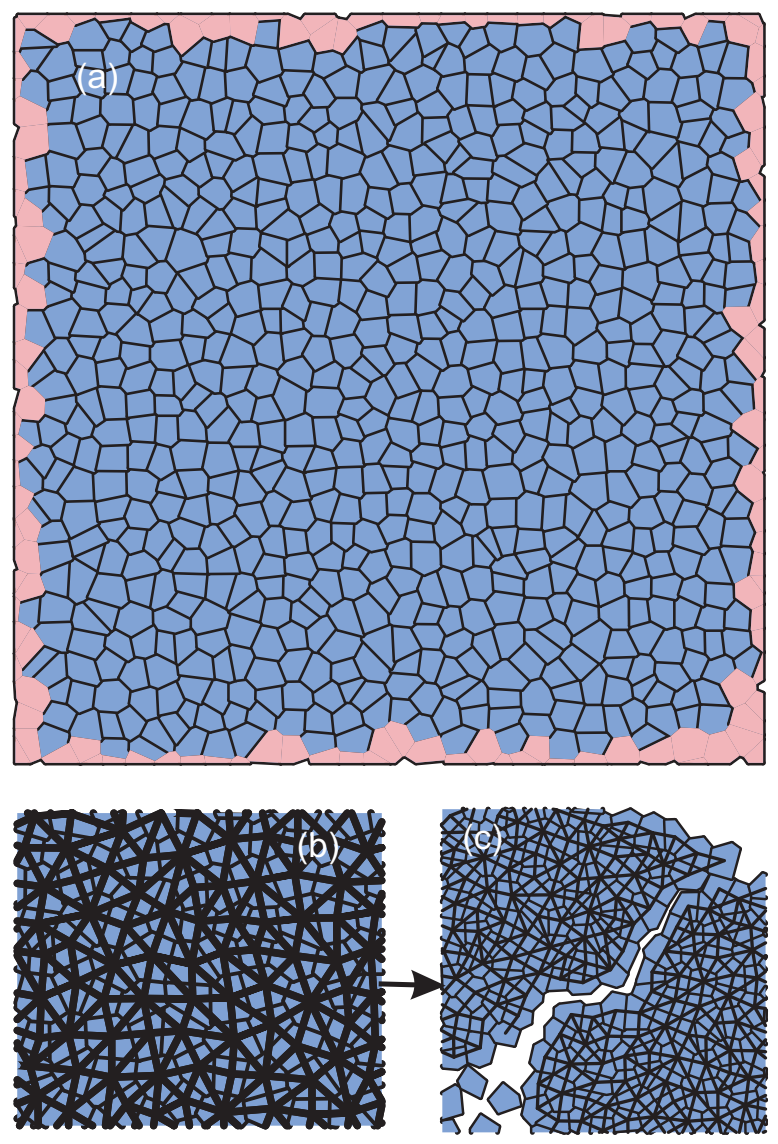

FIG. 1. Demonstration of the model construction for a small system of size $L=30$ : the brittle layer is discretized by means of randomly shaped convex polygons (a). Polygons along the sample perimeter are highlighted by red color. (b) Neighboring polygons are coupled by elastic beams which form a triangular lattice. The polygons are attached to the substrate by spring elements (not shown). (c) The subsequent breaking of beams gives rise to the formation of cracks.

force acting at site $i$ takes the form

$$
F_{n}^{i j}=D_{b}^{i j}\left(u_{n}^{j}-u_{n}^{i}\right),
$$

where the displacement vectors $\vec{u}_{i}$ and $\vec{u}_{j}$ of the two beam ends have the normal and tangential components $\left(u_{n}^{i}, u_{t}^{i}\right)$ and $\left(u_{n}^{j}, u_{t}^{j}\right)[13,15]$.

The shear force is obtained as

$$
F_{t}^{i j}=\beta^{i j}\left(u_{t}^{j}-u_{t}^{i}\right)-\frac{\beta^{i j} l_{0}^{i j}}{2}\left(\Theta^{i}+\Theta^{j}\right),
$$



FIG. 2. Deformation of a single beam in its local coordinate system. The displacement vectors $\vec{u}_{i}, \vec{u}_{j}$ and the bending angles $\Theta^{i}, \Theta^{j}$ are illustrated on the left and right figures, respectively. 
and the flexural torque at site $i$ has the expression

$$
M_{z}^{i}=\frac{\beta^{i j} l^{i j}}{2}\left(u_{t}^{j}-u_{t}^{i}+l^{i j} \Theta^{j}\right)+d^{i j} l^{i j^{2}}\left(\Theta^{j}-\Theta^{i}\right),
$$

which both depend on the displacements $\vec{u}_{i}$ and $\vec{u}_{j}$ and on the bending angles $\Theta^{i}$ and $\Theta^{j}$ of the two beam ends. The elastic constants $D_{b}^{i j}, \beta^{i j}$, and $d^{i j}$ of beams are determined both by the elastic properties of the material and by the geometrical parameters of beams. The value of the elastic constants are calculated in the initial state of the polygon packing as $D_{b}^{i j}=E S_{0}^{i j} / l_{0}^{i j}$, $\beta^{i j}=1 /\left(b^{i j}+c^{i j} / 12\right)$, and $d^{i j}=\beta^{i j}\left(b^{i j} / c^{i j}+1 / 3\right)$, where $b^{i j}=l_{0}^{i j} / G S_{0}^{i j}$, and $c^{i j}=\left(l_{0}^{i j}\right)^{3} / E I^{i j}$. Here $E$ and $G$ are the Young modulus and shear modulus of the material, while $I^{i j}$ denotes the moment of inertia of the beam between sites $i$ and $j$. The value of $b^{i j}$ was set to $b^{i j}=2 D_{b}^{i j}$, which fixes the value of $G[13,15]$. This model has proven successful in reproducing the elastic response of heterogeneous brittle materials under various types of loading conditions $[13,15]$.

To capture the adhesion of the layer to the substrate material the polygons are coupled to the underlaying plane by spring elements. The springs are stress free in the initial position of the polygons $\vec{r}_{i}^{0}=\left(x_{i}^{0}, y_{i}^{0}\right),(i=1, \ldots, N)$; however, they exert a restoring force $\vec{F}_{i}^{s}$ when the polygon gets displaced,

$$
\vec{F}_{i}^{s}=-D_{s}\left(\vec{r}_{i}-\vec{r}_{i}^{0}\right)
$$

Here $D_{s}$ denotes the spring constant characterizing the strength of coupling to the substrate. For simplicity, the same value of $D_{s}$ is used for all polygons without the possibility to break.

When two polygons $i$ and $j$, which are not coupled by beams, come into contact during the breakup process an elastic restoring force $F_{i j}^{c}$ is introduced between them. This contact force is assumed to be proportional to the overlap area $A_{i j}$ and to the Young modulus of the material

$$
\vec{F}_{i j}^{c}=\frac{E A_{i j}}{L_{i j}} \vec{n}_{i j}
$$

Here $L^{i j}$ is a characteristic length of the polygon pair determined as $1 / L_{i j}=1 / R_{i}+1 / R_{j}$, where $R_{i}, R_{j}$ are the radius of circles of the same area as the polygons $[13,15]$. The unit vector $\vec{n}_{i j}$ is perpendicular to the contact line of the polygons (the line connecting the two intersection points) and the force point is placed in the middle of the contact line. Note that this contact force is a generalization of the Hertz contact law for randomly shaped convex objects in two dimensions $[15,18]$. Since the loading condition ensures the opening of cracks, the contact force has solely a minor role in the simulations.

\section{Strain induced by shrinking}

We assume that the thin layer undergoes isotropic shrinking, e.g., due to desiccation while it is attached to the substrate and to the container wall represented by the boundary polygons (see Fig. 1). To capture the effect of shrinking in the model the natural length of beams $l^{i j}$ is gradually decreased as a function of time $t$,

$$
l^{i j}=l_{0}^{i j}(1-s t)
$$

where $s$ denotes the constant shrinking rate. This time evolution gives rise to a uniform shrinkage strain,

$$
\varepsilon=\left(l_{0}^{i j}-l^{i j}\right) / l_{0}^{i j}=s t,
$$

which increases linearly with time. Since the coupling to the substrate and to the side walls prevents the free relaxation stresses build up in the material. To mimic the effect of the container wall, along the external boundary of the sample the particles are fixed, i.e. no displacement and rotation of boundary polygons are allowed. It has the consequence that the uniform shrinkage of beams gives rise to forces and torques on the polygons.

\section{Breaking of cohesive bonds}

To represent the shrinkage-induced breakup of the layer, in the model we assume that solely the beams connecting the particles can break, while the spring elements between the particles and the substrate are not breakable. The breaking of beams is caused by stretching and shear (bending) according to a physical breaking rule,

$$
\left(\frac{\varepsilon_{i j}^{b}}{\varepsilon_{t h}}\right)^{2}+\frac{\max \left(\left|\Theta^{i}\right|,\left|\Theta^{j}\right|\right)}{\Theta_{t h}} \geqslant 1,
$$

where the first and second terms capture the contributions of stretching and bending at the beam ends, respectively [2,13]. Here $\varepsilon_{i j}^{b}$ denotes the local strain of the beam between particles $i$ and $j$. The breaking criterion Eq. (8) is evaluated at each iteration step and those beams which fulfill the condition are removed from the simulations. The subsequent removal of beams leads to the formation of cracks in the layer [see Fig. 1(c)]. Note that the fixed boundary condition, i.e., the coupling to the container wall gives rise to a slight shearing of the beams in spite of the isotropic shrinking.

The value of the breaking parameters $\varepsilon_{\text {th }}$ and $\Theta_{\text {th }}$ control the relative importance of the stretching and bending modes of breaking. To control the amount of disorder in the system both breaking parameters are treated as random variables that are uniformly distributed according to the probability densities,

$$
\begin{gathered}
p\left(\varepsilon_{\mathrm{th}}\right)=1 / 2 W_{\varepsilon}, \\
p\left(\Theta_{\mathrm{th}}\right)=1 / 2 W_{\Theta},
\end{gathered}
$$

over the intervals

$$
\begin{gathered}
\bar{\varepsilon}_{\text {th }}-W_{\varepsilon} \leqslant \varepsilon_{\text {th }} \leqslant \bar{\varepsilon}_{\text {th }}+W_{\varepsilon}, \\
\bar{\Theta}_{\text {th }}-W_{\Theta} \leqslant \Theta_{\text {th }} \leqslant \bar{\Theta}_{\text {th }}+W_{\Theta},
\end{gathered}
$$

around the corresponding average values $\bar{\varepsilon}_{\text {th }}$ and $\bar{\Theta}_{\text {th }}$. The parameters $W_{\varepsilon}$ and $W_{\Theta}$ denote the half width of the range of $\varepsilon_{\text {th }}$ and $\Theta_{\text {th }}$, respectively. Hence, the amount of strength disorder can be characterized by the ratios $\delta_{\varepsilon}=W_{\varepsilon} / \bar{\varepsilon}_{\text {th }}$ and $\delta_{\Theta}=W_{\Theta} / \bar{\Theta}_{\text {th }}$, which can take values between 0 and 1 . In the simulations the same values were used for $\delta_{\varepsilon}$ and $\delta_{\Theta}$, that's why the amount of strength disorder is parametrized by a single value denoted by $\delta$. The disorder distributions are illustrated in Fig. 3 for the tensile mode. 


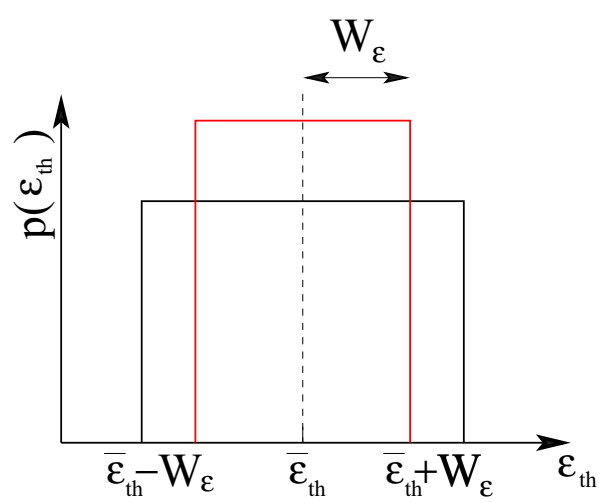

FIG. 3. Probability distribution $p\left(\varepsilon_{t h}\right)$ of the breaking thresholds $\varepsilon_{t h}$ of the stretching breaking mode. Uniform distribution is used where the amount of disorder can be controlled by varying the width $W_{\varepsilon}$. In the figure two distributions of different width are illustrated. The same functional form is considered also for the bending thresholds $\Theta_{\text {th }}$.

It is a very important feature of our model that it contains two sources of disorder: the discretization of the material on a random lattice of convex polygons introduces structural disorder which captures the heterogeneity of the layer at a coarse grained level arising e.g. due to the initial mechanical excitation. The strength disorder is assumed to represent the effect of the local fluctuations of the particle size and of the strength of their cohesive coupling in the material. We performed a large amount of computer simulations varying the amount of strength disorder $\delta$ spanning the entire range of $0 \leqslant \delta \leqslant 1$ at a constant amount of the structural disorder ensured by the constant value of the parameter $a=0.8$.

Discrete element simulations were performed by solving the equation of motion of the polygonal particles by means of a fifth-order Predictor-Corrector scheme [19]. The motion of particles was strongly damped by a velocity dependent friction force. The shrinking rate was set to a low value $s=10^{-5} 1 /$ s so that the damping force was sufficient to suppress oscillations. For each parameter set averages were calculated over 200 simulations with different realizations of disorder. The value of the main parameters of the model are summarized in Table I.

TABLE I. Parameter values used in the simulations.

\begin{tabular}{lcc}
\hline \hline Parameter & & Value \\
\hline Lattice size & $L$ & 80 \\
Number of elements & & 6400 \\
Typical size & & $1.0 \mathrm{~cm}$ \\
of a single element & $\rho$ & $5 \mathrm{~g} / \mathrm{cm}^{3}$ \\
Density & $Y$ & $1 \times 10^{9} \mathrm{dyn} / \mathrm{cm}^{2}$ \\
Elements Young modulus & $E$ & $1 \times 10^{9} \mathrm{dyn} / \mathrm{cm}^{2}$ \\
Beams Young modulus & $D_{s}$ & $5 \times 10^{8}$ \\
Spring constant & $\delta t$ & $1 \times 10^{-6} \mathrm{~s}$ \\
Time step & $s$ & $10^{-5} \mathrm{~s}^{-1}$ \\
Strain rate & $a$ & 0.8 \\
Structural disorder & $\bar{\varepsilon}_{\text {th }}$ & 0.03 \\
Average strength & $\Theta_{\text {th }}$ & $3.0^{\circ}$ \\
Average strength & &
\end{tabular}

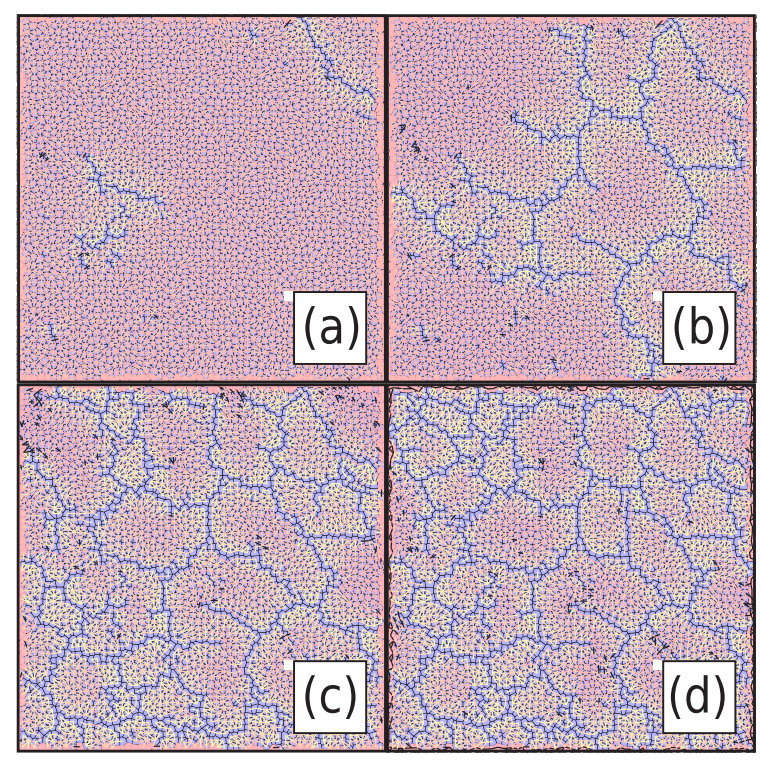

FIG. 4. Time evolution of a system at $\delta=0$. The beams are colored according to their longitudinal strain $\varepsilon$ so that yellow beams are nearly undeformed, while pink and red colors stand for growing elongation and the black beams tend to break. (Blue is the background color of polygons.) Since the strength disorder is zero, only a small amount of random crack nucleation occurs at early stages of the shrinking process (a). As the shrinkage strain $\varepsilon$ increases a connected crack network builds up and the sample falls apart into a large number of pieces $(b, c)$. The fragments undergo further breaking events resulting in usually two daughter pieces (d).

\section{TIME EVOLUTION OF THE FRACTURE PROCESS}

We performed a large amount of computer simulations to obtain a detailed understanding of the dynamics of breakup and time evolution of the shrinking layer. Our main goal is to give a quantitative description of how the statistics of the size of fragments evolves as shrinking proceeds and how this evolution is affected by the amount of disorder of the material. As a representative example in Fig. 4 snapshots of the time evolution are presented for a system at the lowest strength disorder $\delta=0$ where solely the structural heterogeneity of the material controls the behavior of the system. In the figure the beams are colored according to their deformation such that starting from the undeformed state, indicated by yellow color, they gradually get pink, red, and eventually break soon after reaching the black color.

In agreement with former studies on shrinkage-induced cracking [20-22] our simulations revealed that the breakup process of brittle layers has two distinct stages: Before bond breaking sets on the drying-induced shrinkage of beams generates a nearly uniform stress field in the sample such that breaking starts at the weakest bonds. Due to the absence of strength disorder all beams have the same breaking thresholds $\varepsilon_{\text {th }}$ and $\Theta_{\text {th }}$. However, the structural disorder implies a variation of the length and cross section of beams. As a consequence, those beams which are longer and thinner suffer higher a deformation and break easier. The first microcracks nucleate at these weak spots which is then followed by an initiation and rapid growth of cracks [Figs. 4(a) and 4(b)]. This cracking 




FIG. 5. Demonstration of the primary $(\mathrm{a}, \mathrm{b})$ and secondary (c, d) fragmentation mechanisms. The branching-merging scenario of propagating cracks leads to the formation of primary fragments. The tip of the propagating crack in (a) at both ends splits into two subbranches, which then undergo further splitting events. Fragments are created by the merging of nearby subbranches (b). As shrinking proceeds, in the highly stressed interior of fragments a crack emerges and splits the fragment into two pieces (c, d).

process is limited by the relaxation of stress along the crack and by the disorder of the material which can lead to crack arrest. As drying proceeds new cracks nucleate in intact regions and existing cracks undergo growth steps. This damage accumulation goes on until a fully connected crack network emerges along which the layer gets fragmented into numerous pieces; see Figs. 4(c) and 4(d).

Figures 5(a) and 5(b) illustrate that the connected crack network emerges through the branching-merging mechanism: As shrinking proceeds pinned crack tips become unstable and make sudden jumps which in turn can give rise to the splitting of crack tips $[1,20]$. The subsequent splitting events generate treelike crack structures where fragments are formed by the merging of nearby subbranches [see Fig. 5(b)]. In the second stage of the process the primary fragments of the connected crack network further break into smaller pieces [Fig. 4(d)]. This breakup mechanism is demonstrated in Figs. 5(c) and $5(d)$, where the evolution of a single extended fragment can be seen. Along the fragment perimeter the stress is released. However, as shrinking proceeds inside the fragment the strain field gradually builds up which is controlled by the relative stiffness of the lateral beams between polygons and of the coupling to the substrate. Our parameter setup ensures that the highest deformation emerges in the middle of the fragment which results in splitting into two pieces of comparable sizes [Figs. 5(c) and 5(d)] in agreement with former experimental and theoretical studies [1,20,21,23].

\section{EFFECT OF DISORDER ON THE BREAKUP PROCESS}

Simulations showed that the effect of structural disorder can be overcome by a sufficiently high amount of local strength

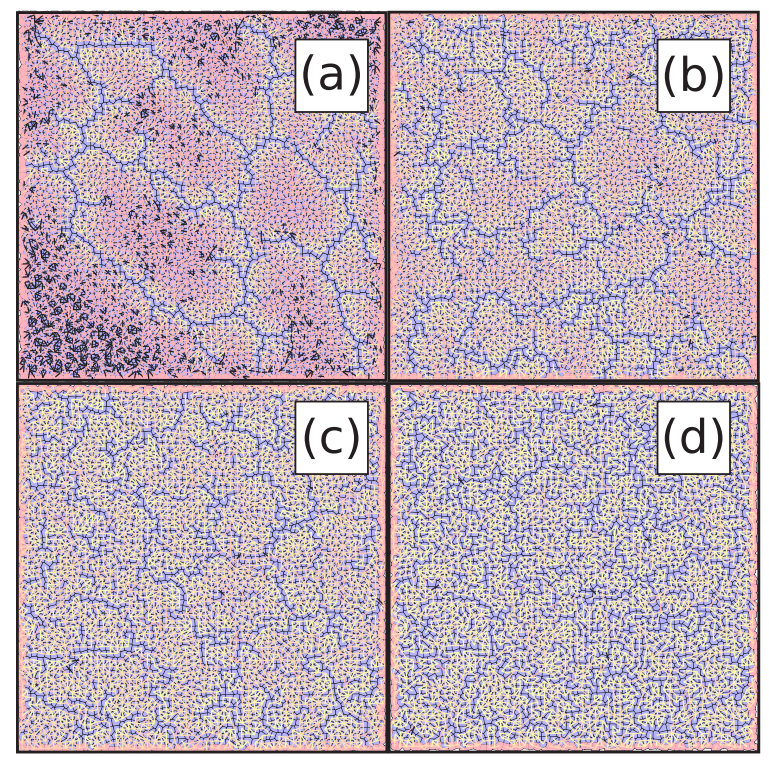

FIG. 6. Snapshot of the system at four different values of strength disorder $\delta=0.0,0.30,0.65,0.80$, for (a), (b), (c), and (d), respectively, obtained at the shrinkage strain where the fully connected crack network is formed.

disorder of beams. We have seen that as shrinkage proceeds damage accumulates in the system; however, the spatial structure of cracks depends on the degree of structural and strength disorder. Figure 6 presents snapshots of the shrinking system at the strain when the connected crack network emerges for four different disorder amplitudes.

For $\delta=0$ the fragments hardly contain internal damage; however, as the strength disorder increases a large amount of diffuse cracks appears, which facilitates the relaxation of the desiccation stress. To give a quantitative characterization of this damage accumulation Fig. 7 shows the fraction of broken beams $n_{b}$, i.e., the number of broken beams $N_{\mathrm{bb}}$ normalized by the total number of beams $N_{\mathrm{bt}}$ initially present in the system

$$
n_{b}=\left\langle N_{\mathrm{bb}} / N_{\mathrm{bt}}\right\rangle
$$

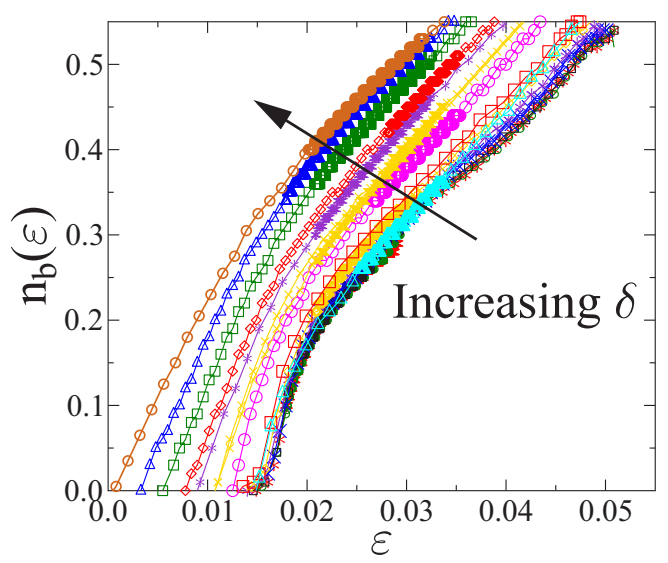

FIG. 7. The fraction of broken beams $n_{b}(\varepsilon)$ as a function of strain $\varepsilon$ for several values of the amount of disorder $\delta$, from left to right $\delta=$ $1.0,0.9,0.8,0.7,0.6,0.5,0.4,0.3,0.25,0.2,0.15,0.1,0.05,0.0$. 


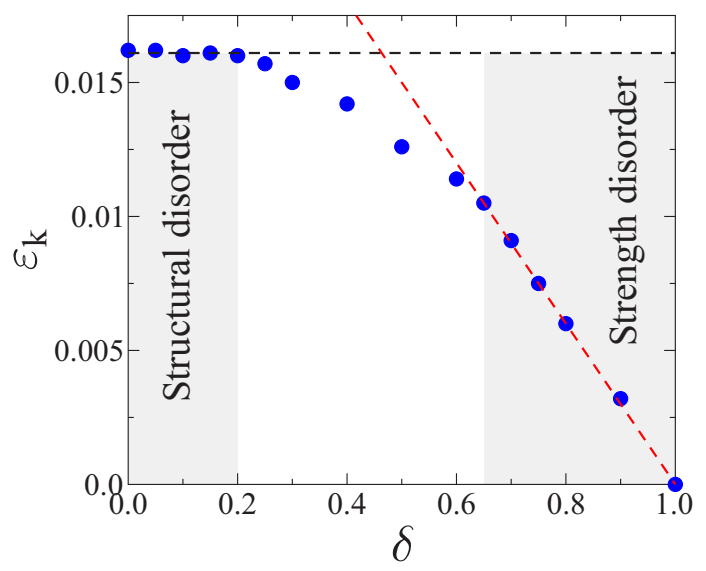

FIG. 8. The threshold strain $\varepsilon$ where cracking sets on in the layer varying the amount of strength disorder in the entire range $0 \leqslant \delta \leqslant 1$.

as a function of shrinkage strain $\varepsilon$. The $n_{b}(\varepsilon)$ curves are shown for several values of the amount of disorder $\delta$, in each case averaging over 200 simulations with different realizations of disorder. It can be observed in all cases that for breaking to occur the strain has to surpass a threshold value $\varepsilon_{k}(\delta)$, which is determined by the weakest bonds. Of course, $n_{b}(\varepsilon)$ must be a monotonically increasing function; however, its precise form is affected by both disorder sources. For the highest disorder level $\delta=1$ the distribution of breaking thresholds spans down to zero strength so that in Fig. 7 cracking starts already at the very beginning of shrinking $\varepsilon_{k}(\delta=1)=0$. Reducing the amount of disorder $\delta$ the onset of cracking becomes more-and-more abrupt, i.e., $\varepsilon_{k}$ shifts to higher values followed by a rapid raise of the $n_{b}(\varepsilon)$ curves. As the limit of zero strength disorder is approached $\delta \rightarrow 0$ the functional form of $n_{b}$ tends to a limit curve. If strength disorder dominates the primary crack nucleation the value of $\varepsilon_{k}(\delta)$ should be equal to the lowest strength value,

$$
\varepsilon_{k}(\delta)=\bar{\varepsilon}_{t h}(1-\delta) .
$$

We determined numerically the value of $\varepsilon_{k}$ which is presented in Fig. 8 as a function of $\delta$. Good agreement with Eq. (14) can be observed in the parameter range $\delta \geqslant 0.65$, which determines the high disorder regime of the breaking process. On the contrary, for the range $\delta \leqslant 0.20$ the onset strain of cracking proved to be independent of $\delta$ which marks the dominance of structural disorder. In between a broad transient regime emerges where the competition of two disorders results in a complex accumulation of damage.

We note that in Fig. 7 the fraction of broken beams is practically the cumulative probability of breaking $P_{b}(\varepsilon)$, which in principal could be obtained analytically from the density functions Eq. (10). Neglecting the role of bending in breaking, a linear function is obtained $P_{b}(\varepsilon)=\left(1 / 2 W_{\varepsilon}\right)\left(\varepsilon-\bar{\varepsilon}_{t h}+W_{\varepsilon}\right)$ for $\varepsilon \geqslant \bar{\varepsilon}_{t h}-W_{\varepsilon}$. It can be observed in the figure that close to the onset of cracking the $n_{b}(\varepsilon)$ curves are linear in agreement with the above arguments. However, the starting slope is higher than $1 / 2 W_{\varepsilon}$, which shows that the shearing of beams plays a role in the crack formation.

Our calculations were performed at a single value of the amount of structural disorder $a$, where $a$ was chosen to provide

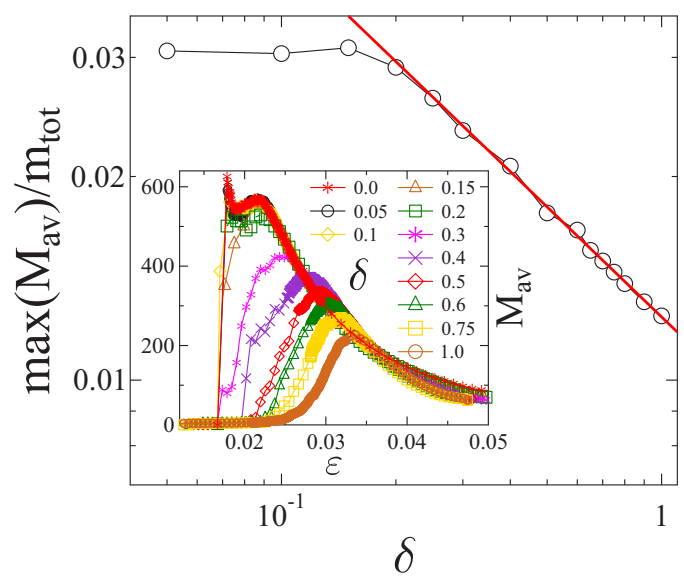

FIG. 9. Main panel: Maximum of $M_{\text {av }}$ normalized by the total mass $m_{\text {tot }}$ of the system as a function of the disorder amplitude on a double logarithmic plot. The straight line represents a power law of exponent 0.54 . Inset: The average fragment mass $M_{\mathrm{av}}$ as a function of strain $\varepsilon$ for several values of the disorder amplitude $\delta$.

a Voronoi lattice of polygons and a triangular lattice of beams with a high degree of isotropy. When the amount of structural disorder $a$ is reduced the lattice structure converges to a regular square lattice, where all the beams have the same geometrical and physical properties. As a consequence, the dominance of strength disorder win Fig. 8 gradually expands down to $\delta=0$ with a linear behavior of $\varepsilon_{k}(\delta)$ in Eq. (14) over the entire $0 \leqslant \delta \leqslant 1$ interval.

\section{TRANSITION TO FRAGMENTATION}

Shrinkage-induced cracking gives rise to the formation of a connected crack network, where closed loops of cracks enclose fragments and results in the breakup of the layer into a large number of pieces. Before the emergence of the spanning crack network the system is damaged; however, it practically keeps its integrity. To characterize how this transition from the damaged to the fragmented state occurs during the desiccation process we determined the average mass of fragments $M_{a v}$ as a function of strain $\varepsilon$. For a single sample the average fragment mass was obtained as the ratio of the second $M_{2}$ and first $M_{1}$ moments of fragment masses $m_{i}, i=1, \ldots, K$, where $K$ denotes the total number of fragments in the layer at a given $\varepsilon$. The $q$ th moment of the fragment ensemble is defined as

$$
M_{q}=\sum_{i=1}^{K} m_{i}^{q},
$$

where the ' indicates that the largest fragment mass $M_{\max }$ is skipped from the summation. Then $M_{\text {av }}$ was obtained by averaging the value of $M_{2} / M_{1}$ over a large number of samples

$$
M_{\mathrm{av}}=\left\langle M_{2} / M_{1}\right\rangle .
$$

It can be observed in the inset of Fig. 9 that the $M_{\mathrm{av}}(\varepsilon)$ curves have a well-defined maximum the position of which depends on the disorder amplitude $\delta$. For high disorder $\delta \rightarrow 1$ fragment formation starts early, but these fragments are much smaller than the original size of the system. Since the largest fragment is always omitted in the calculation of moments, 
the position of the maximum $\varepsilon_{c}$ marks the point where the crack network gets connected and spans the entire system so that the dominating fragment suddenly breaks up into a large number of pieces [24]. Beyond the maximum no dominating fragment exists, and all fragments undergo gradual breakup as shrinking proceeds. It follows that depending on the value of the shrinkage strain $\varepsilon$ the system has two phases, i.e., damaged and fragmented with a transition point at $\varepsilon_{c}$. Of course, the critical strain $\varepsilon_{c}$ of fragmentation is greater than the onset strain of cracking $\varepsilon_{c}(\delta)>\varepsilon_{k}(\delta)$. However, with decreasing $\delta$ the position of the maximum $\varepsilon_{c}$ shifts downward in the inset of Fig. 9 approaching the value of $\varepsilon_{k}$; furthermore, the rise of the $M_{\text {av }}$ curves get sharper indicating that the breaking process becomes more and more abrupt.

It is interesting to note that with increasing disorder amplitude $\delta$ the maximum of $M_{\text {av }}$ decreases. The result shows that at higher disorder a larger fraction of bonds breaks until the critical point is reached (see also Fig. 7), which gives rise to smaller fragments when the spanning crack network emerges. To quantify this effect we determined the value of the maximum $\max \left(M_{\mathrm{av}}\right)$ as a function of $\delta$. It can be observed in Fig. 9 that for low strength disorder the maximum converges to a limit value while for higher disorder a power-law decrease is obtained,

$$
\max \left(M_{\mathrm{av}}\right) \sim \delta^{-\gamma}
$$

Best fit was obtained with the exponent $\gamma=0.54(3)$. Comparing to Fig. 8 it is remarkable that the upper limit of the dominance of structural disorder falls close to the value $\delta \approx 0.2$ obtained from the onset of cracking. However, the scaling regime extends both to the strength disorder and transient regimes.

\section{FRAGMENT MASS DISTRIBUTIONS}

It has been discussed that during the drying process the size of fragments continuously change, i.e., even beyond the fragmentation critical point $\varepsilon_{c}$ fragments undergo breakup events reducing their size. When structural disorder dominates, the process is binary breakup, while for high strength disorder the coalescence of smaller cracks is the dominating mechanism of size reduction.

To get a quantitative insight into the evolution of fragment masses, Fig. 10 presents the mass distribution $p(m, \varepsilon)$ of fragments obtained at several different strain values above the fragmentation critical point $\varepsilon>\varepsilon_{c}$ at zero-strength disorder. The distributions are asymmetric with a long tail at all shrinkage stages $\varepsilon$; however, with increasing $\varepsilon$ both the average and the scatter of mass values decrease. Figure 11 demonstrates that rescaling the mass distributions with the average mass of fragments $\langle m\rangle$ the $p(m, \varepsilon)$ curves of different $\varepsilon$ can be collapsed on a master curve. The high-quality data collapse implies the scaling structure of the distributions,

$$
p(m, \varepsilon)=\langle m\rangle^{-1} \Psi(m /\langle m\rangle),
$$

where $\varepsilon$ dependence only occurs through the arithmetic average of fragment masses $\langle m\rangle(\varepsilon)$. The scaling function $\Psi(x)$

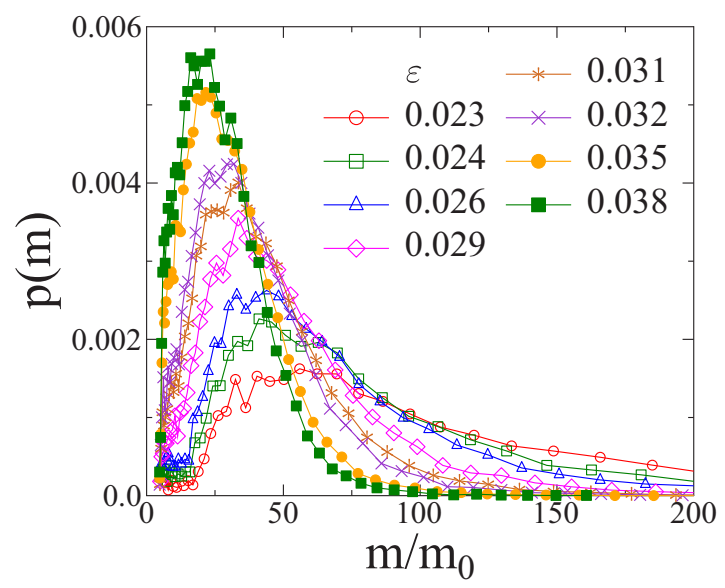

FIG. 10. Mass distribution of fragments $p(m)$ measured at different strain values $\varepsilon$ in the fragmentation phase at zero strength disorder $\delta=0.0$. The corresponding critical point of fragmentation is $\varepsilon_{c}=0.017$. The fragment mass $m$ is made dimensionless by division with the average mass of single polygons $m_{0}$.

can be very well described by the log-normal distribution

$$
\Psi(x)=\frac{1}{x \sigma \sqrt{2 \pi}} \exp \left[-(\ln (x)-\mu)^{2} / 2 \sigma^{2}\right],
$$

where $\mu$ and $\sigma$ denote the average and the standard deviation, respectively. The continuous line in Fig. 11 presents a fit of the numerical data with Eq. (19). Deviations from Eq. (19) can be observed in the regime of small fragment masses due to the existence of unbreakable fragments (single polygons). The log-normal mass distribution is consistent with the binary splitting of the fragments which gives rise to a cascade process of fragment creation [21,25].

The above functional form and scaling structure of $p(m, \varepsilon)$ proved to be valid until the cracking process is dominated by the structural disorder. Our computer simulations revealed that increasing the amount of strength disorder the mass



FIG. 11. Data collapse of the mass distributions of fragments obtained by rescaling the two axis of Fig. 10 with the arithmetic average of fragment masses. The continuous line represents a fit with the log-normal distribution Eq. (19). Note the logarithmic scale on the horizontal axis. 


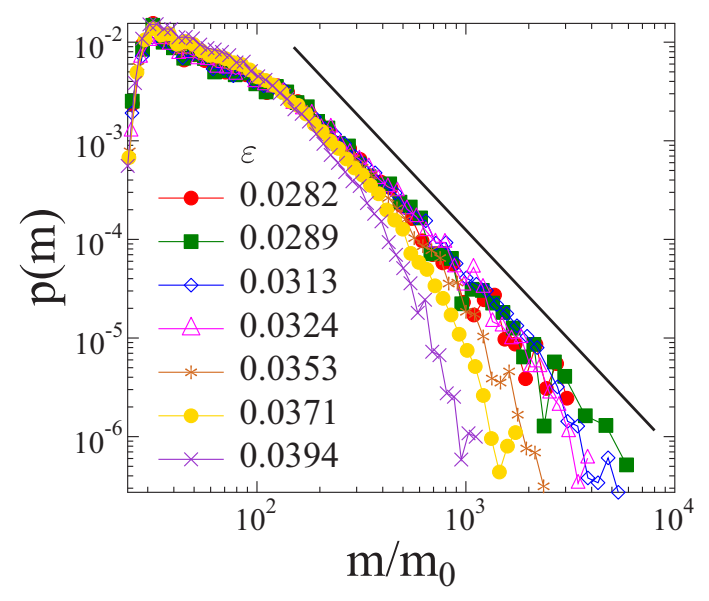

FIG. 12. Mass distribution of fragments at high strength disorder $\delta=0.8$ for several different $\varepsilon$ values. The straight line represents a power law of exponent $\tau=2.25$. The fragment mass $m$ is made dimensionless by division with the average mass of single polygons $m_{0}$.

distribution gets gradually transformed into a power law followed by an exponential cutoff. This is illustrated in Fig. 12 at $\delta=0.8$ for several values of $\varepsilon$ in the fragmented regime $\varepsilon>\varepsilon_{c}$. The main effect of the increasing strain is that the cutoff mass of the distributions shifts to lower values. In the vicinity of the critical point the functional form of $p(m, \varepsilon)$ can be well described by the expression

$$
p(m, \varepsilon) \propto m^{-\tau} \exp \left(-m / m_{c}\right)
$$

where strain dependence only occurs through the cutoff mass $m_{c}(\varepsilon)$. Careful scaling analysis showed that $m_{c}$ is not a homogeneous function of strain, hence, rescaling the data with powers of $\varepsilon$ no collapse could be achieved. This may be caused by the system size, i.e., the lattice size of the simulations was not sufficient to reach the scaling regime. The value of the exponent $\tau$ of the power-law regime was determined as $\tau=2.25(4)$, which implies a rapid decrease of the frequency of fragments with their mass. The power-law functional form is the consequence of the gradual coalescence of randomly nucleated cracks, which is the dominating breakup mechanism in the limit of high-strength disorder [2,24,25].

\section{DISCUSSION}

We constructed a discrete element model of shrinkageinduced cracking of a thin brittle layer coupled to a substrate. The key feature of the model is that it has two sources of disorder, i.e., structural and strength disorder controlled by the parameters $a$ and $\delta$, respectively. The model is not fitted to any specific type of material. However, in the case of drying dense colloidal suspensions structural and strength disorder of the model may represent different scale heterogeneities caused, e.g., by an inherent initial deformation field imprinted in the paste layer by mechanical excitations and by the variation of the size of colloidal particles. The competition of these two effects has been found to play a key role in the emergence of the memory effect in desiccation-induced cracking of paste layers $[1,6,7,12]$.
Our computer simulations revealed that shrinkage-induced cracking has two phases: at low-shrinkage strains the layer gets damaged, however, it retains its integrity, while for high-shrinkage strains it fragments into a large number of pieces. The transition between the damaged and fragmented states occurs at a critical strain where a connected crack network emerges along which the layer falls apart.

The relative amount of the two disorders proved to be essential for the cracking mechanism and for the statistics of fragments. At low-strength disorder the branching-merging scenario is the governing mechanism of the network formation of cracks, while at high-strength disorder the coalescence of randomly nucleated cracks dominates. Once the spanning crack network has been formed fragments undergo sequential breakup due to binary splitting for low disorder, leading to a log-normal distribution of fragment masses. For increasing disorder the nucleation and coalescence play an increasing role which transforms the mass distribution into a power law with an exponential cutoff.

It is interesting to note that the branching-merging dynamics of cracks has been found before to be responsible for the power-law mass distribution of pieces in the dynamic fragmentation of heterogeneous brittle materials [26-29]. In the dynamic case branching occurs due to the Yoffe instability of high speed cracks, while for shrinking thin layers crack jumps are moderated by the underlying substrate which gives more room for the materials disorder. In the high-disorder limit of the model the random nucleation and coalescence of cracks is essentially a percolation mechanism, which also explains why the power-law distribution of fragment masses is obtained.

Recently, a smoothed particle hydrodynamics model of paste layers has been introduced to study the desiccationinduced cracking process [21,22]. The mass distribution of fragments was found to have a functional form close to a lognormal distribution, however, with a right-handed asymmetry. The authors proved that the binary breakup of fragments can be described as a Gibrat-process where the size-dependent lifetime of fragments is responsible for the deviation from the log-normal form [22]. In our discrete element model the existence of unbreakable particles gives rise to the distortion of the mass distribution which becomes apparent in the limit of structural disorder.

Our study demonstrates that the controlled competition of structural and strength disorder in a discrete element framework is a promising approach to investigate the memory effect of desiccation-induced crack patterns in pastes $[1,6,7]$. In the present study we have considered isotropic structural disorder; however, initial mechanical vibration and flow imprint anisotropic plastic deformation fields in pastes. As to the next, this effect will be captured in our model by introducing anisotropy into the underlying random lattice of polygonal elements.

\section{ACKNOWLEDGMENTS}

This work was supported by Project No. K 119967 and by the JSPS-HAS collaborative Grant No. NKM-101/2015 MTA-JSPS project. 
[1] L. Goehring, A. Nakahara, T. Dutta, S. Kitsunezaki, and S. Tarafdar, Desiccation Cracks and Their Patterns: Formation and Modelling in Science and Nature (John Wiley \& Sons, New York, 2015).

[2] H. J. Herrmann and S. Roux, eds., Statistical Models for the Fracture of Disordered Media, Random materials and processes (Elsevier, Amsterdam, 1990).

[3] A. Groisman and E. Kaplan, Europhys. Lett. 25, 415 (1994).

[4] H. Nahlawi and J. K. Kodikara, Geotech. Geol. Eng. 24, 1641 (2006).

[5] S. Nag, S. Sinha, S. Sadhukhan, T. Dutta, and S. Tarafdar, J. Phys.: Cond. Matt. 22, 015402 (2010).

[6] A. Nakahara and Y. Matsuo, J. Stat. Mech.: Theor. Exp. (2006) P07016.

[7] A. Nakahara and Y. Matsuo, Phys. Rev. E 74, 045102 (2006).

[8] K. H. Nam, I. H. Park, and S. H. Ko, Nature 485, 221 (2012).

[9] L. Guo, Y. Ren, L. Y. Kong, W. K. Chim, and S. Y. Chiam, Nat. Commun. 7, 13148 (2016).

[10] H. Nakayama, Y. Matsuo, O. Takeshi, and A. Nakahara, Eur. Phys. J. E 36, 1 (2013).

[11] Y. Matsuo and A. Nakahara, J. Phys. Soc. Jpn. 81, 024801 (2012).

[12] S. Kitsunezaki, A. Nakahara, and Y. Matsuo, Europhys. Lett. 114, 64002 (2016).

[13] F. Kun and H. J. Herrmann, Comp. Meth. Appl. Mech. Eng. 138, 3 (1996).
[14] F. Kun and H. J. Herrmann, Phys. Rev. E 59, 2623 (1999).

[15] G. A. D'Addetta, F. Kun, and E. Ramm, Gran. Matt. 4, 77 (2002).

[16] B. Behera, F. Kun, S. McNamara, and H. J. Herrmann, J. Phys.Cond. Mat. 17, 2439 (2005).

[17] K. B. Lauritsen, H. Puhl, and H.-J. Tillemans, Int. J. Mod. Phys. C 5, 909 (1994)

[18] T. Pöschel and T. Schwager, Computational Granular Dynamics (Springer, Berlin, 2005).

[19] M. P. Allen and D. J. Tildesley, eds., Computer Simulation of Liquids (Oxford University Press, Oxford, 1984).

[20] S. Kitsunezaki, Phys. Rev. E 60, 6449 (1999).

[21] S.-i. Ito and S. Yukawa, Phys. Rev. E 90, 042909 (2014).

[22] S.-i. Ito and S. Yukawa, J. Phys. Soc. Jap. 83, 124005 (2014).

[23] T. Hornig, I. M. Sokolov, and A. Blumen, Phys. Rev. E 54, 4293 (1996).

[24] D. Stauffer and A. Aharony, Introduction to Percolation Theory (Taylor \& Francis, London, 1992).

[25] J. A. Aström, Adv. Phys. 55, 247 (2006).

[26] P. Kekalainen, J. A. Aström, and J. Timonen, Phys. Rev. E 76, 026112 (2007).

[27] J. Åström and J. Timonen, Phys. Rev. Lett. 78, 3677 (1997).

[28] J. A. Aström, F. Ouchterlony, R. P. Linna, and J. Timonen, Phys. Rev. Lett. 92, 245506 (2004).

[29] F. K. Wittel, F. Kun, H. J. Herrmann, and B. H. Kröplin, Phys. Rev. Lett. 93, 035504 (2004). 